\title{
Introduction to the special issue on quantum imaging
}

\author{
Robert W. Boyd · Peter J. Reynolds
}

Received: 16 March 2012 / Accepted: 19 March 2012 / Published online: 29 March 2012

(C) Springer Science+Business Media, LLC 2012

Advances in both quantum optics and in quantum information science that took place through the 1990's opened the possibility of entirely new methods for forming (and even storing) images, some with unprecedented sensitivity and resolution. This new field of research, known as Quantum Imaging, led to other conceptual breakthroughs as well, such as the possibility of imaging without interaction. Moreover, quantum imaging exploits quantum parallelism in ways intrinsic to image-bearing beams, leading to large information capacity. Significant progress in the conceptual understanding of both image formation and of quantum protocols has resulted from recent research in quantum imaging.

Quantum imaging also holds great promise for addressing practical, real-world problems. In view of this potential, a major research initiative was proposed in 2003 at the Army Research Office to create a so-called MURI-a Center (delocalized, but nevertheless logistically a Center) — to explore further this emerging field of Quantum Imaging. The initiative was successful, and a competition was launched in 2004. The winning Center was chosen to be at the University of Rochester, with

\author{
R. W. Boyd $(\varangle)$ \\ Institute of Optics, University of Rochester, Rochester, NY 14627, USA \\ e-mail: boydrw@mac.com \\ R. W. Boyd \\ Department of Physics and Astronomy, University of Rochester, Rochester, NY 14627, USA \\ R. W. Boyd \\ Department of Physics and School of Electrical Engineering and Computer Science, \\ University of Ottawa, Ottawa, ON K1N 6N5, Canada \\ P. J. Reynolds \\ Physics Division and Physical Sciences Directorate, US Army Research Office, \\ Research Triangle Park, NC 27709, USA
}


Robert Boyd as the Director. The total membership of the Center included: University of Rochester (Robert Boyd and John Howell), Boston University (Malvin Teich, Alexander Sergienko, and Bahaa Saleh), Louisiana State University (Jonathan Dowling), MIT (Jeffrey Shapiro), Northwestern University (Prem Kumar and Geraldo Barbosa), and University of Maryland, Baltimore County (Yanhua Shih, Morton Rubin, and Fow-Sen Choa). The principal research themes of the Center have been (i) ghost imaging, (ii) quantum lithography, (iii) quantum optical-coherence tomography, (iv) quantum laser radar, (v) single-photon imaging, and (vi) interactionfree imaging.

Many of the research "themes" listed above have played a key role in the development more generally of the field of quantum imaging. Briefly, the essence of each of them is this: (i) Ghost imaging exploits coincidence techniques to form images using photons that have never interacted with the object to be imaged. (ii) Quantum lithography is based on quantum-entangled photons that can write structures at a resolution exceeding that imposed by classical diffraction theory. (iii) Quantum optical coherence tomography uses quantum effects to increase the axial resolution of the imaging system, and to extract useful information regarding the dispersion of the material. (iv) Quantum laser radar allows for a noise-free quantum preamplifier that can increase the sensitivity of detection. (v) Single-photon imaging provides the ability to store and recall an entire image encoded in a single photon. (vi) Interaction-free imaging allows for sensing of objects without any photons actually interacting with the object to be sensed. Distinct from ghost imaging, this is, in effect, a way to make an "invisible quantum tripwire."

In achieving the goals of the Center, not just science but new technologies were developed. Examples of technologies that have played a key role in the development of quantum imaging include the creation of intense sources of entangled photons based upon (a) guided-wave interactions in periodically poled materials, (b) third-order interactions in atomic vapors, and (c) the orbital angular momentum of light beams. Also important has been the development of means of producing high-order entanglement, both in the sense of two-photon entanglement in a large Hilbert space of pixels and in the sense of entanglement of more than two photons. Both experimental and theoretical studies of these issues have been conducted.

This special issue consists of four review articles deriving from the work of the Center. They span many of the topics of current interest in the field of Quantum Imaging. However, not all areas are covered-not even all areas that were worked on in the Center. Nevertheless, the essence of what was done by the Center is here. Boyd and Dowling present a review of the field of quantum lithography, including the choice of material systems for its implementation. Teich, Saleh, Wong, and Shapiro present a review of the field of quantum optical coherence tomography. Howell, Anisimov, Dowling, and Boyd present a review of imaging modalities based on the use of individual (or small numbers of) photons and biphotons. They also give a review of high-dimensional quantum communication. This contribution includes a discussion of interaction-free imaging, as well as discussion of image storage with a single photon. Finally, Shapiro and Boyd present a review of the physics of ghost imaging. There are a number of different ghost imaging modalities. Some require a fully quantum description while others can be described semiclassically. Shih presents 
a Comment on ghost imaging, espousing the view that lensless pseudothermal ghost imaging in particular must be described fully quantum mechanically. There has been ongoing debate in the literature on this point. Here we hope we put the issue to rest by presenting Shih's argument in its clearest essence, followed by a reply from Shapiro and Boyd. We believe that the short clear exposition here, presented in one place, side by side, should finally lay this issue to rest.

The articles that comprise this Special Issue represent the core of the progress achieved in Quantum Imaging resulting from the MURI Center described, with research that spanned from 2005 through 2011. We hope the reader comes away with an appreciation of the excitement, progress, and potential in this field. 